\title{
Optical vector analyzer for characterization of Fano resonance structures based on unbalanced double-sideband modulation
}

\author{
Oleg Morozov*, Airat Sakhabutdinov, Ilnur Nureev, and Samvel Papazyan \\ Kazan National Research Technical University named after A.N. Tupolev-KAI, \\ Radio photonics and Microwave Technologies Department, \\ 10 Karl Marx str., 420111, Kazan, Tatarstan, Russian Federation
}

\begin{abstract}
We report an optical vector analyzer (OVA) for characterization of Fano resonance structures based on unbalanced optical double-sideband (UB-ODSB) carrier modulation, with capability to investigate the optical structures with asymmetric and ultra-narrowband spectral responses. The ratio of UB-ODSB sidebands amplitudes is not equal to one and constant throughout the measurement process. Transmitted through the optical resonant structure UB-ODSB-radiation is sent to photodetector, that is used to convert the optical signal to electrical one. Output photodetector signal contains the beat components of the UBODSB-radiation sidebands and optical carrier. These components splits up by bandpass filters (BPF) for post-processing of measurement results in accordance with new mathematical model that is also presented in this paper. The proposed approach allows to measure phase and magnitude responses of the optical device under test (ODUT) with high resolution; to formulate a criterion for signal falling within the limits of the investigated spectral response; to take one-step measurements.
\end{abstract}

\section{Introduction}

The theoretical investigations of the ring fiber structures with the $\pi$-phase-shifted fiber Bragg grating (FBG) have been carried out over the past five years [1]. It is proved that the spectrum of this structure is asymmetric and has two components. It consists of the left component, corresponding to the symmetric Lorentz resonance, and the right asymmetric component, corresponding to the Fano resonance. The Fano component has a narrower passband and high Q-factor, which is much higher than the characteristics of ordinary ring resonators and linear $\pi$-phase-shifted FBGs.

Undoubtedly, the use of Fano resonance from this point of view provides a powerful stimulus in the development of sensory systems, but it actualizes the problem of controlling the shape of the spectral response, determining its center frequency, maximum gain and qfactor at various levels of the spectral response due to its asymmetry.

* Corresponding author: microoil@mail.ru 
The optical vector analyzers (OVAs), which allows measuring the magnitude and phase responses of investigated resonant structures, or another optical devices under test (ODUTs), solve these problems. The problem of controlling the spectral shape of an asymmetric and ultra-narrowband resonant structures imposes stringent requirements on the accuracy of measurements and the resolution of OVAs, which have been consistently improved during the development of these devices.

The basis of first OVAs were phase-shift modulation and interferometric methods, which involved scanning the frequency responses by the wavelength-swept laser source [2]. The disadvantage of these approaches is the low resolution of hundreds of $\mathrm{MHz}$, which makes such devices inapplicable for the analysis of narrowband spectral responses.

Significant progress in this area is associated with the advent of OVAs based on optical single-sideband modulation (OSSB) [3-6]. The main advantage of this method is its high resolution that reach up to $78 \mathrm{kHz}$ in [6] and realized due to the possibility of linearly changing the frequency of scanning in units of $\mathrm{Hz}$ and potentially sub- $\mathrm{Hz}$. These devices made it possible to investigate narrowband spectral responses, but at the same time has serious disadvantages: higher-order sidebands in the OSSB-signal spectra, the intensity of which increases with increasing of modulation index, and small first sideband suppression ratio. The beat components of the undesired first and higher-order sidebands on the photodetector with the frequency of modulation signal causes errors into the measurement results, and the higher their intensity, the grosser the error. Therefore, it is necessary to limit the modulation index, but it not eliminate the error, and only reduces it [5]. Since such OSSB modulators are hard to be achieved, the performance, especially the measurement range, accuracy and dynamic range, of the OSSB-based OVA is restricted [7].

The solution of the above problems led to the creation of OVAs based on optical double-sideband modulation (ODSB). Such devices are divide into two types - with shifted optical carrier [7-11] and with asymmetry in spectrum components amplitudes [12-16].

The main problem of the first method is to generate ODSB-signal with optical carrier, which is asymmetrically located respect to the scanning sidebands [7-9]. When this signal received by photodetector, the frequencies of beat components of the shifted optical carrier and scanning sidebands will be different from the modulation frequency and not equal to each other. It should be noted, that the formation of the required ODSB-signal with shifted carrier is also a difficult task, so these OVAs needs into further simplification, for example, as in $[10,11]$. The main problem of the second method is the introduction of amplitude asymmetry for the components of the ODSB signal using different devices [12-14] and measurements in two stages on different components. Depending on the implementation of the method, either the amplitudes of the sidebands or the amplitude of the carrier for each measurement stage may differ. The calculation of the magnitude response and phase response of ODUTs on two interfering components with different amplitudes requires sufficient complex calculations, the use of complex calibration techniques and high stability of the measuring circuit, since the calculations relate to amplitude measurements. However, this method is simpler in the required scanning ODSB-signal generation process $[15,16]$.

We report an OVA for characterization of resonance structures based on unbalanced optical double-sideband modulation (UB-ODSB), with capability to investigate the optics structures with asymmetric and ultra-narrowband spectral responses, such as Fano resonance. The key element of the device is a UB-ODSB optical radiation source, which consist of a narrowband laser source, two sequentially connected Mach-Zehnder modulators with optical bandpass filter (OBPF) between them. The modulators are configured so that first of it is ODSB-modulator with suppressed carrier (ODSB-SC). So the initial single-frequency optical carrier is suppressed in it, but with forming a symmetric ODSB-radiation, one sideband of which lies in the investigated frequency range. The linear sweeping of this sideband (measuring optical carrier - MOC) sweeps it optical radiation inside ODUTs spectrum. OBPF is suppressed second sideband and not fully suppressed 
initial carrier. The second modulator is the OSSB-modulator. We use all disadvantages of OSSB in useful manner. The modulation frequency of the second modulator is constant, so bandpass problems of $90^{\circ}$ phase shifter is eliminated. It selected so in units of $\mathrm{kHz}$ range or smaller, that the sidebands are located so close to the MOC that the transmission coefficient of the ODUTs for all components of the UB-ODSB-radiation spectrum one can take as the same. The undesired sideband specially is not fully suppressed (another problem of OSSBmodulators is eliminated) and formed amplitude asymmetry, so sideband suppression ratio in modulator is not large and not equal to one. In other words, at any scanning frequency, at any point of the ODUTs, the relative change in the amplitudes of the spectral components of the scanning signal can be considered the same with a sufficient degree of accuracy. Thus, during the scanning process, the ratio of the amplitudes of the MOC and sidebands of the scanning radiation remains constant, which, as will be shown, allows to restore the shape of the investigated resonance structure with high resolution.

Transmitted through the ODUT UB-ODSB-radiation is sent to photodetector, that is used to convert the optical radiation to photocurrent. It contains the beat components of the UB-ODSB sidebands and MOC: DC-component and AC-components with modulation and doubled modulation frequencies. These components splits up respectively by electronic low pass filter (ELPF) and bandpass ones (EBPFs) for post-processing of measurement results in accordance with a proposed new mathematical model, which allows to get a frequency responses of the ODUT. The resolution of OVA is defined by initial carrier line width, and additionally by law of frequency scanning in first modulator and value of modulation frequency of second one. The main advantage of proposed OVA is one-step measurements.

\section{OVA block-diagram}

Block-diagram of the proposed OVA based on UB-ODSB modulation is shown on Fig. 1.

Single-frequency laser diode (LD) radiation with an angular frequency $\omega_{0}$ sent to the first Mach-Zehnder ODSB-SC-modulator. The radio frequency generator RF1 is a source of modulation signal with frequency of MOC $\omega$. Since ODSB-SC-modulator operates at the zero point of the modulation characteristic, the optical carrier and even-order sidebands of amplitude-modulated signal at the modulator output are suppressed. This radiation can be considered as an ODSB-radiation with suppressed optical carrier (point A). Then OBPF with independently tunable central wavelength, bandwidth and attenuation, selects one of the optical sidebands, suppresses another one and remained part of initial carrier (point B). The radio frequency generator RF2 is a source of modulation signal for OSSB-modulator with frequency of $\Omega$, which transfer on it through $90^{\circ} \mathrm{HC}$. Since second modulator operates as OSSB-modulator with not fully suppressed undesired sideband at the linear section of the modulation characteristic, both spectral components are existed, but sideband suppressed ratio is not equal to one (point $\mathrm{C}$ ). The modulation frequency of the second modulator is constant and selected so in units of $\mathrm{kHz}$ range or smaller, that the sidebands are located so close to the MOC that the transmission coefficient of the ODUTs for all components of the UB-ODSB-radiation spectrum one can take as the same.

The resulting UB-ODSB-radiation passes through isolator and SMF to the ODUT or reference channel (RC) without it. It scans ODUT spectral response by sweeping the MOC frequency of generator RF1. The output photocurrent of PD1 (point D) contains the beat components of the UB-ODSB-modulation sidebands and MOC: DC-component and ACcomponents with modulation $\Omega$ and doubled modulation $2 \Omega$ frequencies. These components splits up respectively by ELPF и EBPF1, EBPF2 filters for post-processing of measurement results in PMD and PU.

The RC is used to OVA calibration and phase measurements. 

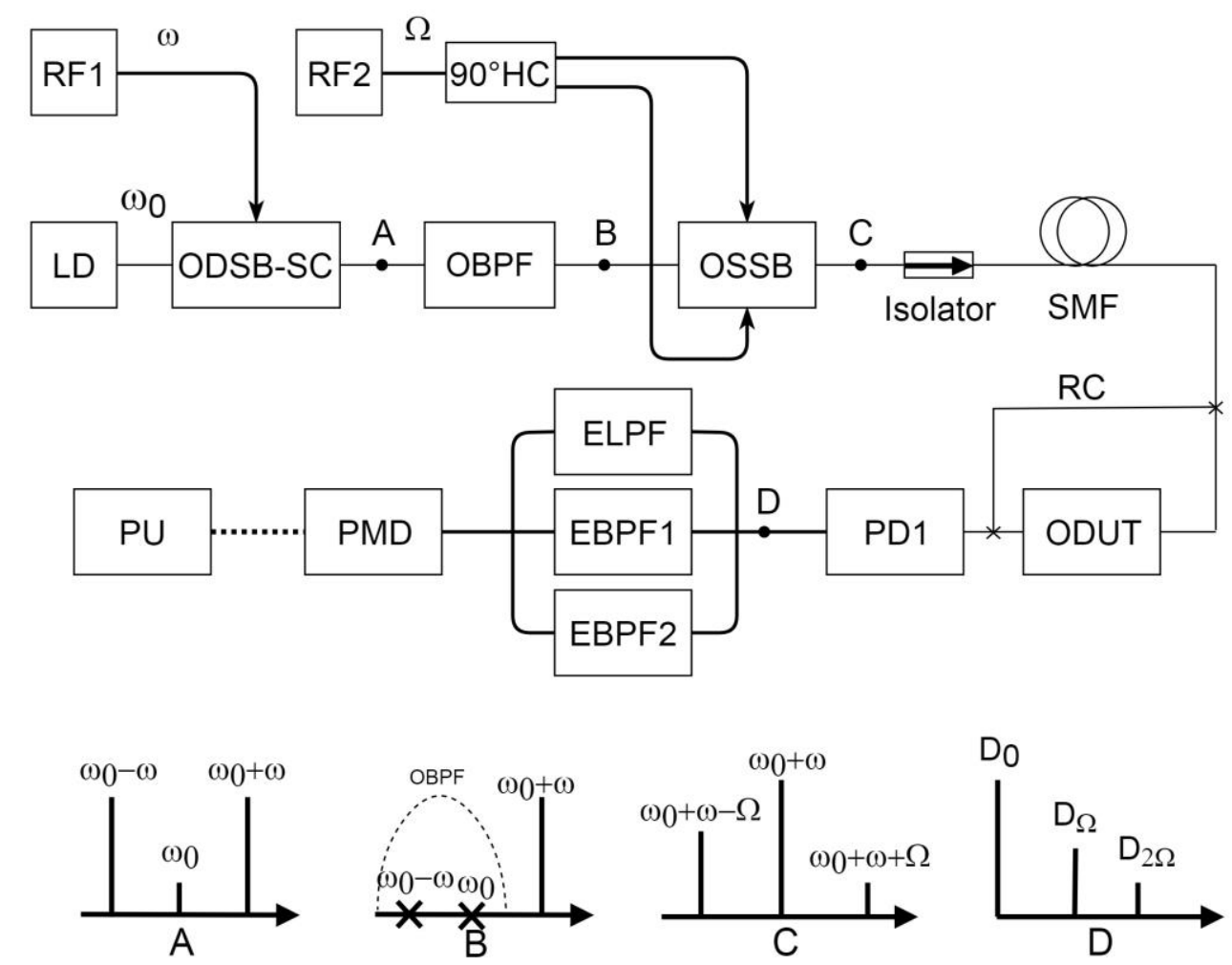

Fig.1. Block-diagram of the proposed OVA based on UB-ODSB modulation: LD - laser diode; ODSB-SC - Mach-Zehnder amplitude one-drive ODSB-SC-modulator; OSSB - Mach-Zehnder amplitude dual-drive OSSB-modulator; RF1 - radiofrequency generator for MOC sweeping; RF2 - radiofrequency generator with constant frequency for unbalanced radiation forming; HC - $90^{\circ}$ hybrid coupler; OBPF - optical bandpass filter; SMF - single-mode fiber; ODUT - optical device under test; RC - reference channel; PD - photodetector; ELPF - electronic low pass filter; EBPF1 - electronic bandpass filter on $\Omega$; EBPF2 - electronic bandpass filter on $2 \Omega$; PMD - phasemagnitude detector; PU - process unit; thin lines - optical signal; bold lines - electrical signal.

The RC is used to OVA calibration and phase measurements.

\section{Mathematical model}

The optical output result of UB-ODSB-radiation and the ODUT interaction can be written as the sum of its three spectral components:

$$
\begin{gathered}
S(t)=A \cdot C_{0 L} \cdot C_{L}(\omega) \cdot \sin \left((\omega-\Omega) t+\phi_{L}(\omega)\right)+ \\
+A \cdot C_{0 C} \cdot C_{C}(\omega) \cdot \sin \left(\omega t+\phi_{C}(\omega)\right)+A \cdot C_{0 R} \cdot C_{R}(\omega) \cdot \sin \left((\omega+\Omega) t+\phi_{R}(\omega)\right)
\end{gathered}
$$

where $A$ is the amplitude of MOC; $C_{0 L}, C_{0 C}, C_{0 R}$ are the constant coefficients, which determine the known relation of spectral components amplitudes of the left, central, and right spectral components with the indices $L, C$, and $R$ respectively; $C_{L}(\omega), C_{C}(\omega)$ and $C_{R}(\omega)$ are functions of frequency and determined by the ODUT spectral characteristic; $\omega-$ $\Omega, \omega, \omega+\Omega$ are the instantaneous frequencies of the UB-ODSB-radiation of the left, central, and right components respectively; $\varphi_{L}(\omega), \varphi_{C}(\omega)$ and $\varphi_{R}(\omega)$ describe the phase change of the UB-ODSB-radiation after it pass through the ODUT. 
The resulting radiation is received at the photodetector, which is a nonlinear lowfrequency element and the resulting output current of the photodetector is proportional to the square of the input radiation. Thus, the photocurrent at the output of the photodetector can be expressed as:

$$
\begin{gathered}
I(t) \sim A_{L}^{2}+A_{C}^{2}+A_{R}^{2}+2 A_{C} A_{L} \cdot \cos \left(\Omega t+\left(\phi_{C}+\phi_{L}\right)\right)+ \\
+2 A_{C} A_{R} \cos \left(\Omega t+\left(\phi_{R}-\phi_{C}\right)\right)+2 A_{L} A_{R} \cos \left(2 \Omega t+\left(\phi_{R}-\phi_{L}\right)\right)
\end{gathered} .
$$

In order to simplify recording and mathematical transformations in expression (2), we used an implicit form of recording the phases dependence on frequency: $\varphi_{L}=\varphi_{L}(\omega-\Omega)$, $\varphi_{C}=\varphi_{C}(\omega), \varphi_{R}=\varphi_{R}(\omega+\Omega)$, and amplitudes of spectral components on frequency: $A_{L}=A_{L}(\omega)=A \cdot \mathrm{C}_{0 L} \cdot \mathrm{C}_{L}(\omega), A_{C}=A_{C}(\omega)=A \cdot \mathrm{C}_{0 C} \cdot \mathrm{C}_{C}(\omega), A_{R}=A_{R}(\omega)=A \cdot \mathrm{C}_{0 R} \cdot \mathrm{C}_{R}(\omega)$.

Having installed three frequency filters after the photodetector: at a DC power level $D_{0}$, AC components at the modulation frequency $D_{\Omega}$ and double modulation frequency $D_{2 \Omega}$, we obtain a system of three equations for determining six unknowns:

$$
\left\{\begin{array}{l}
D_{0}=A_{L}^{2}+A_{C}^{2}+A_{R}^{2} ; \\
D_{\Omega}=2 A_{C} \sqrt{A_{L}^{2}+A_{R}^{2}+2 A_{L} A_{R} \cos \left(2 \phi_{C}-\phi_{L}-\phi_{R}\right)} \\
D_{2 \Omega}=2 A_{L} A_{R} .
\end{array}\right.
$$

The unknown quantities in (3) are $A_{L}, A_{C}, A_{R}$ - three amplitudes of the UB-ODSBradiation components and $\phi_{L}, \phi_{C}, \phi_{R}$ - phase delays at the same frequencies.

The system (3) cannot be solved because it contains too many unknown quantities. This difficulty can be overcome applying the following technique, the essence of which is as follows. We assume that the doubled modulation frequency $2 \Omega$ will be less than the required scan detail. Under these conditions, we can assume that the ratio of the amplitudes of UBODSB-signal components is a constant before and after it passes through the resonant structure. In addition, the phases of the left and right components of the UB-ODSB-radiation can be equated to the phase of the optical carrier with a high degree of accuracy. Given the above simplifications and returning to explicitly writing the dependence of the amplitudes on the frequency, the system of equations (3) will take the form (4):

$$
\left\{\begin{array}{l}
D_{0}=A_{L}^{2}(\omega)+A_{C}^{2}(\omega)+A_{R}^{2}(\omega) \\
D_{\Omega}=2 A_{C}(\omega)\left(A_{L}(\omega)+A_{R}(\omega)\right) \\
D_{2 \Omega}=2 A_{L}(\omega) A_{R}(\omega)
\end{array}\right.
$$

Based on these assumptions, it is possible to search for the amplitude of the ODUT at the scanning frequency, which, in fact, will determine the magnitude response of the device under test. Having solved the system of equations (4), we obtain the equation (5) which describes the amplitude of the ODUT at the scanning frequency:

$$
A_{C}(\omega)=H_{O D U T}(\omega)=\sqrt{\frac{1}{2} \frac{C_{0 C}}{C_{0 L}} \frac{C_{0 C}}{C_{0 R}} D_{2 \Omega}(\omega)} .
$$

To determine the phase response, it is necessary to sum two signals, from passed through the ODUT and through the $\mathrm{RC}$ respectively. The signal passed through the $\mathrm{RC}$ can be written as:

$$
\begin{gathered}
B(t, \omega)=A_{L}(\omega) \sin \left((\omega-\Omega) t+\phi_{0}\right)+ \\
A_{C}(\omega) \sin \left(\omega t+\phi_{0}\right)+A_{R}(\omega) \sin \left((\omega+\Omega) t+\phi_{0}\right)
\end{gathered}
$$


and the signal passed through the ODUT:

$$
\begin{gathered}
S(t, \omega)=A_{L}^{\prime}(\omega) \sin \left((\omega-\Omega) t+\phi_{0}+\psi(\omega)\right) \\
+A_{C}^{\prime}(\omega) \sin \left(\omega t+\phi_{0}+\psi(\omega)\right)+A_{R}^{\prime}(\omega) \sin \left((\omega+\Omega) t+\phi_{0}+\psi(\omega)\right)
\end{gathered} .
$$

The output current of the photodetector will be proportional to the square of the sum of signals (6) and (7):

$$
R(t, \omega)=(B(t, \omega)+S(t, \omega))^{2} .
$$

By squaring and simplifying expression (8), after a series of transformations, the phase response can be obtained:

$$
\psi(\omega)=\arcsin \left(\frac{1}{2} \frac{\left.D(t, \omega)\right|_{2 \Omega}}{A_{L}^{\prime}(\omega) A_{R}(\omega)-A_{L}(\omega) A_{R}^{\prime}(\omega)}\right) .
$$

Thus, the calculations performed by equations (5) and (9) in the data processing unit allow to restore the magnitude and phase responses of the device under test.

According to this model, the criterion for all UB-ODSB-radiation components fall into the frequency range of the investigated ODUT can be written as:

$$
\begin{gathered}
C r_{1}\left(\frac{C_{0 L}}{C_{0 R}}\right)=\frac{D_{0}(\omega)}{D_{2 \Omega}(\omega)}=\frac{1}{2}\left(\frac{C_{0 L}}{C_{0 R}}+1+\frac{C_{0 R}}{C_{0 L}}\right) \equiv \text { const, } \\
C r_{2}\left(\frac{C_{0 L}}{C_{0 R}}, \frac{C_{0 C}}{C_{0 R}}\right)=\frac{D_{\Omega}(\omega)}{D_{2 \Omega}(\omega)}=2\left(\frac{C_{0 C}}{C_{0 R}}+\frac{C_{0 C}}{C_{0 L}}\right) \equiv \text { const. }
\end{gathered}
$$

Outside the investigated ODUT, there is no practically light response from it. Consequently, the amplitudes of the constant signal level and oscillations at the modulation and doubled modulation frequencies will be extremely close to zero. The ratio of two quantities extremely close to zero gives in the general case is an arbitrary value. Note that the right-hand sides in (10) are known quantities determined by the ratio of the amplitudes of UB-ODSB-radiation components. We conclude that if the first or second expressions obtained in the course of measurements in (10) are not equal to the previously known constants, then the UB-ODSB-radiation components does not fall within a limits of ODUT. Directly from here follows one more conclusion. If during measurements, the absolute value of criterions $\mathrm{Cr}_{1}$ or $\mathrm{Cr}_{2}$ in (10) differs from the known values of the constants by an amount greater than a predetermined error, this serves as a requirement to reduce the modulation frequency of UB-ODSB-radiation.

\section{Experimental results}

An experiment based on the setup shown in Fig. 1 is carried out. Initial carrier radiation is formed by DFB-1550-14BF laser with a central wavelength of $1546.55 \mathrm{~nm}$ and controlled by the Pilot 4AC driver. UB-ODSB-radiation source consists from two modulators made by PNPPK (Perm) with half-wave voltage 3,2 V and bandwidth 11-13 GHz and OBPF Finisar WaveShaper 4000S (with independently controlled central wavelength, passband and transmission coefficient). The modulation signal is generated by the Agilent $5701 \mathrm{C}$ electronic vector analyzer (EVA), and its frequency is determined by the conditions for the required measurement accuracy. The resulting scanning radiation passes through the FBG Fano resonance structure (ODUT), and is received by a PR-20-D photodiode with a bandwidth of $20 \mathrm{GHz}$. ELPFs and EBPFs from Micran Corp. process the appropriate 
frequencies of the output photodetector signal. Output components are compared in an EVA, which is connected to a microcontroller (personal computer), which allows get and display the obtained frequency responses of the FBG Fano resonance structure.

Firstly, it is necessary to find the frequency range of the ODUT. This problem can be solved by using the developed criterions (10). By linearly modulating the MOC frequency, the frequency range of ODUT spectral response can be found. If the signal falls within the limits of the resonance structure, expressions (10) are equal to predetermined constants, which corresponds to flat section of the curve in Fig. 2. If the signal does not fall within the limits of the investigated resonance structure, expressions (10) take arbitrary values, which corresponds to the ripples shown in Fig. 2.

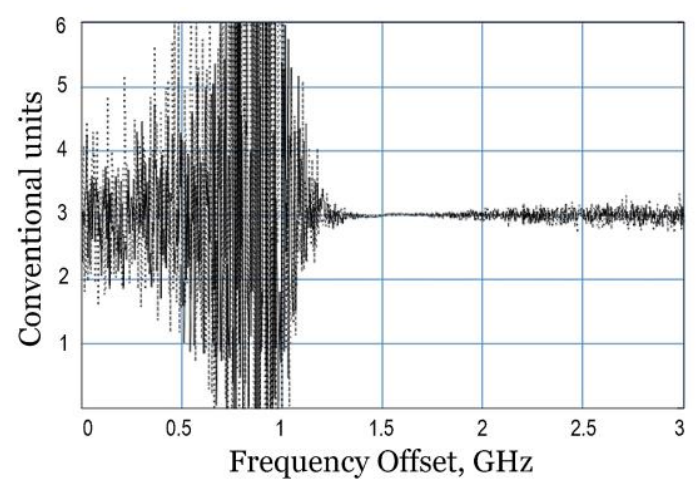

Fig. 2. Fulfillment of the criterions (10) for UB-ODSB-radiation fall into the frequency range of the ODUT.

Figure 2 shows that the investigated spectral response is located in the frequency offset range from $1.3 \mathrm{GHz}$ to $1.75 \mathrm{GHz}$, therefore, measurements are carried out in this frequency range. The measurement results are shown in Fig. 3, where effective points are obtained over a span of $450 \mathrm{MHz}$ with resolution of $128 \mathrm{kHz}$. A magnitude of $5.5 \mathrm{dBm}$ and a bandwidth of $200 \mathrm{MHz}$ are observed.

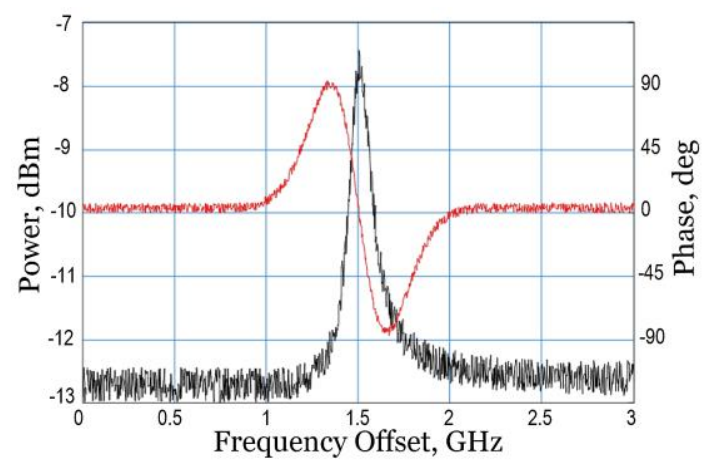

Fig. 3. Frequency responses of FBG Fano resonance structure: black line - magnitude response; red line - phase response.

\section{Conclusion}

A new OVA based on UB-ODSB-modulation was proposed, modelled and experimentally demonstrated. It allows to carry out one-step measurements of ultra-narrowband and asymmetric resonant structures such as Fano resonance, with the remaining simplicity of 
obtaining the UB-ODSB-radiation. The proposed OVA based on new mathematical model, which describes the methodology of measurements and signal processing after photodetector. In addition, it allows to get the criterion for all UB-ODSB-radiation components fall into the frequency range of the investigated resonant structure. The basic principle of the mathematical model is that the sidebands of the UB-ODSB-radiation are so close to the carrier that the transmission coefficient of the resonant structure can be considered as the same for all spectral components with a sufficient degree of accuracy. The new approach allows to investigate the magnitude and phase responses of different narrow band optical structures, but not limited by them.

Ministry of Science and Higher Education of Russian Federation (8.6872.2017/8.9, program "Asymmetry") supported this work.

\section{References}

1. C. E. Campanella, F. De Leonardis, L. Mastronardi, P. Malara, G. Gagliardi, V. M. N. Passaro, Opt. Express, 23, 14301-14313 (2015)

2. G. D. VanWiggeren, A. R. Motamedi, D. M. Baney, IEEE Photon. Technol. Lett., 15, 263-265 (2003)

3. J. E. Román, M. Y. Frankel, and R. D. Esman, Opt. Lett., 23, 939-941 (1998)

4. Sh. Pan, M. Xue, 12th International Conference on Optical Communications and Networks (IEEE, Chengdu, 2013)

5. M. Xue, Sh. Pan, D. Zhu, Y. Zhao, 12th International Conference on Optical Communications and Networks (IEEE, Chengdu, 2013)

6. Z. Z. Tang, S. L. Pan, J. P. Yao, Opt. Express, 20, 6555-6560 (2012)

7. T. Qing, Sh. Li, M. Xue, W. Li, N. Zhu, Sh. Pan, Opt. Express, 25, 4665-4671 (2017)

8. T. Qing, M. Xue, M. Huang, Sh. Pan, Opt. Lett., 39, 6174-6176 (2014)

9. T. Qing, S. Li, M. Xue, Sh. Pan, Opt. Lett., 41, 3671-3674 (2016)

10. O. G. Morozov, I. I. Nureev, A. Zh. Sakhabutdinov, R. Sh. Misbakhov, S. Papazyan, 2019 Systems of Signals Generating and Processing in the Field of on Board Communications (IEEE, Moscow, 2019)

11. O. G. Morozov, I. I. Nureev, A. Zh. Sakhabutdinov, R. Sh. Misbakhov, T. R. Sakhbiev, R. Nurullin, S. Papazyan, L. M. Sarvarova, Proc. SPIE, 11146, 111460R (2019)

12. M. Xue, S. Liu, Sh. Pan, IEEE Phot. Tech. Lett., 30, 491-494 (2018)

13. W. Jun, L. Wang, C. Yang, M. Li, N. H. Zhu, J. Guo, L. Xiong, W. Li, Opt. Lett., 42, 4426-4429 (2017)

14. M. Wang, J. Yao, IEEE Phot. Techn. Lett., 25, 753-756 (2013)

15. O. G. Morozov, G. A. Morozov, I. I. Nureev, D. I. Kasimova, M. Yu. Zastela, P. V. Gavrilov, I. A. Makarov, V. A. Purtov, Proc. SPIE, 9807, 980717 (2016)

16. T. R. Sakhbiev, O. G. Morozov, I. I. Nureev, A. J. Sakhabutdinov, L. M. Faskhutdinov, 2018 Systems of Signal Synchronization, Generating and Processing in Telecommunications (IEEE, Minsk, 2018) 